\title{
Levels of Acid Sphingomyelinase (ASM) in Caenorhabditis elegans in Microgravity
}

Annabel K. Gravely ${ }^{1 *}$, Alice Vlasov ${ }^{1 *}$, Amy Freeman ${ }^{1, *}$, Kay Wu ${ }^{1,{ }^{*}}$, Nathaniel J. Szewczyk ${ }^{2}$, Robert $\mathrm{D}^{\prime} \mathrm{Cruz}^{3}$, and Jane Batt ${ }^{3}$

\author{
${ }^{1}$ University of Toronto Schools, Toronto, Ontario; ${ }^{2}$ University of Nottingham, MRC/Arthritis Research UK Centre \\ for Musculoskeletal Ageing Research \& National Institute for Health Research (NIHR) Nottingham Biomedical \\ Research Centre, Royal Derby Hospital Centre, Derby, Derbyshire; ${ }^{3}$ Keenan Research Center for Biomedical \\ Sciences, St. Michael's Hospital, Toronto, Ontario $\quad{ }^{*}$ Co-primary authors
}

\begin{abstract}
Both Amyotrophic Lateral Sclerosis (ALS) patients and astronauts in spaceflight suffer from muscle atrophy. Previous research suggests that the enzyme acid sphingomyelinase (ASM) may be involved in the pathogenesis of ALS, but it is not known if ASM influences muscle atrophy in microgravity. In this study, Caenorhabditis elegans (C. elegans) were exposed to microgravity conditions on the International Space Station (ISS) within the confines of a Fluid Mixing Enclosure (FME). Return of the FME yielded 72,050 live nematodes, the first demonstration of $C$. elegans survival of space travel in an FME. After the nematodes returned to
\end{abstract}

Key words: Muscle Atrophy; Student Spaceflight Experiments Program (SSEP); Fluid Mixing Enclosure System (FME); International Space Station (ISS); C. elegans; Sphingomyelinase; Amyotrophic Lateral Sclerosis (ALS)

Correspondence to: Jane Batt

St. Michael's Hospital

30 Bond Street

Toronto, Ontario

Canada

M5B 1W8

Telephone: 416-864-5412

E-mail: jane.batt@utoronto.ca
Earth, in much larger numbers than seen in previous FME experiments, the size and ASM expression levels in experimental worms were compared to control Earth-bound worms. $C$. elegans that returned from the ISS were larger in both length and cross-sectional area than the control worms, and they exhibited decreased expression of ASM-1 and ASM-2 proteins. Further research must be conducted to elucidate the role of ASM in muscle atrophy, as there were many limitations to this study. Understanding the role of ASM in muscle atrophy may lead to the discovery of novel targets for treatment of both ALS and muscle atrophy in microgravity. This study was a student led initiative and undertaken as a project within the Student Spaceflight Experiments Program (SSEP), under the auspices of the National Center for Earth and Space Science Education and the Arthur C. Clarke Institute for Space Education.

\section{INTRODUCTION}

Amyotrophic Lateral Sclerosis (ALS) is a neurodegenerative disease characterized by a loss of motor neurons and, as a result, skeletal muscle atrophy (van Es et al., 2017). Muscle atrophy is the decrease in muscle size/mass that results from loss of contractile and structural proteins and muscle cells (Brooks and Myburgh, 2014; Fanzani 
et al., 2012). The progressive motor neuron loss, denervation-induced and primary muscle dysfunction and wasting in ALS culminates in paralysis and death (van Es et al., 2017; Loeffler et al., 2016). Unfortunately, there are currently no effective curative treatments or therapeutic strategies for ALS (van Es et al., 2017).

Muscle atrophy is prevalent across many chronic diseases (Powers et al., 2016a), in individuals subjected to prolonged bedrest and immobility (Brooks and Myburgh, 2014), and in astronauts in spaceflight (Fitts et al., 2010). In fact, the neuromuscular system is seen as one of the most heavily impacted physiological systems in microgravity (Fitts et al., 2001). Muscle atrophy in microgravity has been previously linked to a lack of exercise opportunities (Fitts et al., 2010). However, the use of resistive exercise countermeasures programs by astronauts was only able to partially mitigate muscle atrophy in microgravity (Fitts et al., 2010; Tanaka et al., 2017). Given the engineering challenge to generate artificial gravity in spaceflight, and the inability of resistance exercise to prevent atrophy in microgravity, it is essential to continue to delineate the molecular mechanisms underpinning muscle loss in space to enable the development of effective interventions to preserve and/or restore muscle mass. We were provided an opportunity by the Student Spaceflight Experiments Program (SSEP), a space education program aimed at students from elementary school to college level administered through the National Center for Earth and Space Science Education (NCESSE) and the Arthur C. Clarke Institute for Space Education, to conduct an experiment on the International Space Station (ISS). We decided to investigate the underlying causes of muscle atrophy in microgravity. This research may bring us one step closer to developing an effective treatment for muscle wasting and weakness in both astronauts during spaceflight and in individuals with chronic diseases such as ALS.

Oxidative stress contributes to the development of skeletal muscle atrophy in both chronic disease states and inactivity via a myriad of cellular signaling networks (Cutler et al., 2002; Powers et al., 2016b), Microgravity has been shown to induce oxidative stress (Takahashi et al., 2017). Sphingomyelin and ceramide accumulation have been linked to both ALS pathogenesis and to oxidative stress (Cutler et al., 2002). Furthermore, in the $\mathrm{SOD}^{\mathrm{G} 86 \mathrm{R}}$ ALS mouse model, there is evidence of increased gene expression of some sphingomyelinasesenzymes that catalyzes the hydrolysis of sphingomyelin to phosphorylcholine and ceramide (Henriques et al., 2018). This could contribute further to ceramide accumulation. Acid sphingomyelinase (ASM) levels are elevated and activity is increased in the presence of oxidative stress, infection, and inflammation (Kornhuber et al., 2015). However, ASM also releases ceramide, which in itself induces oxidative stress (Jana et al., 2009). These data together suggest that ASM dysregulation may be involved in the pathology of ALS and muscle wasting of spaceflight

We therefore undertook a study to determine ASM levels in Caenorhabditis elegans (C. elegans), a small, yet complex organism with a neuromuscular system: (Szewczyk and Jacobson, 2005). C. elegans is a small nematode that is very similar genetically to humans (Altun and Hall, 2006). In fact, almost $40 \%$ of its genes are closely related to those of humans and $C$. elegans is recognized as a suitable model for studying human disease in space (Adenle et al., 2009). $C$. elegans has three ASMs, but this study focuses on ASM-1 and ASM-2. Both ASM-1 and ASM-2 are $30 \%$ homologous to the human ASM (Lin et al., 1998).

In this study, C. elegans were sent to the ISS to experience microgravity, while control $C$. elegans remained on Earth. We hypothesized that the worms on the ISS would develop muscle atrophy and experience increased ASM levels compared to the ground experiment. Atrophy extent was determined by morphometric analyses. ASM expression was determined by measuring protein levels. Overall, this study aimed to elucidate the role of ASM in muscle atrophy in microgravity, which may have additional implications for diseases characterized by muscle disuse and wasting.

\section{MATERIALS AND METHODS}

\section{Housing and Feeding of C. elegans}

This experiment was conducted within a Type 3 Fluids Mixing Enclosure (FME) system (NanoRacks, Houston, TX, USA), designed for space research. FMEs have been used for all 
previous SSEP experiments (Warren et al., 2013). As depicted in Figure 1, the FME is a flexible silicone tube, divided into three $2.8 \mathrm{~mL}$ compartments by plastic clamps. Sterilization of the FME minilab was achieved by autoclaving the tube at $121^{\circ} \mathrm{C}$ for 20 minutes, spraying the caps and clamps with $70 \%$ ethanol followed by exposure to ultraviolet (UV) light for 1 hour.

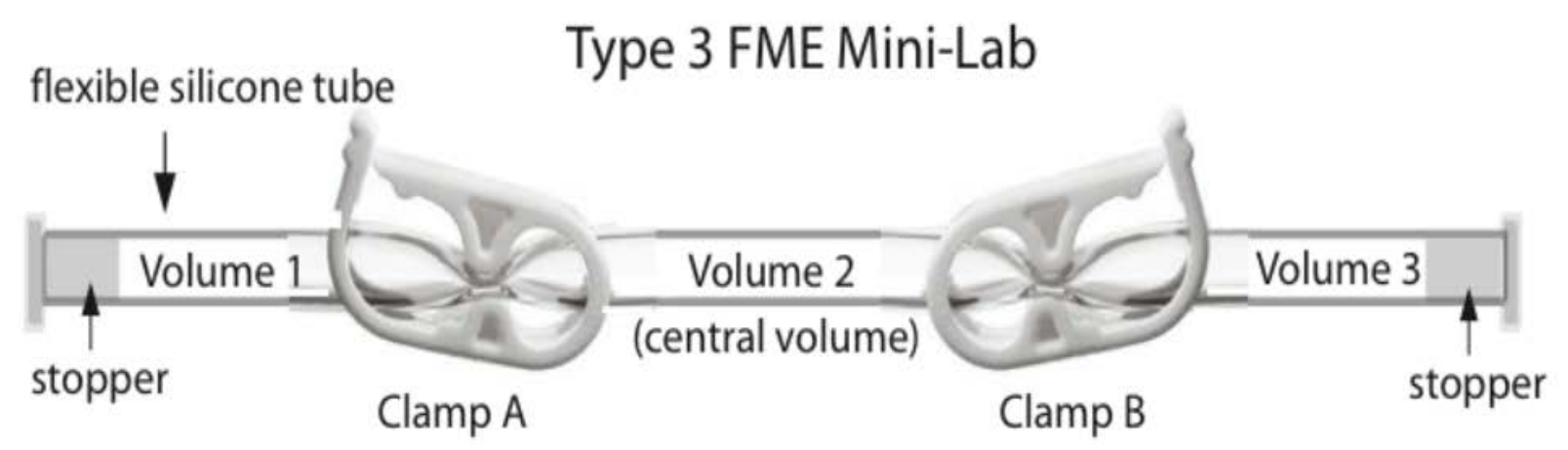

(Total Volume $=8.4 \mathrm{ml}=$ Volume $1+$ Volume $2+$ Volume 3 )

Figure 1. Type 3 Fluids Mixing Enclosure (FME) Mini-Lab. The FME is $17 \mathrm{~cm}$ in length with a total volume of $8.4 \mathrm{~mL}$ divided into three $2.8 \mathrm{~mL}$ compartments. Volume 1 and 3 contained $2.4 \mathrm{~mL}$ of CeMM and Volume 2 contained $2.4 \mathrm{~mL}$ of CeMM housing 5,000 C. elegans.

C. elegans maintenance medium (CeMM, a gift from Dr LR. Garcia, Howard Hughes Medical Ins, Texas A\&M University) was used as the food source and growth medium in the FME. CeMM was used instead of E. coli, the traditional food source for C. elegans (Avery and You, 2012), because $E$. coli is viewed as a toxic risk on the ISS. $2.4 \mathrm{~mL}$ of CeMM was added to each of Chambers 1, 2, and 3 of the FME. In Chamber 2, 5,000 C. elegans were added to the $2.4 \mathrm{~mL}$ of CeMM. This compartmentalization ensured that the amount of food available to the worms could be controlled and rationed by unclamping and reclamping the tube. The worms loaded were at mixed stages of development, which helped ensure their survival under the unpredictable conditions during pre-flight storage and shipment (Szewczyk, 2005). The FME systems were assembled at room temperature in the ground laboratory. They were sealed on both ends with plastic caps, twist ties, and gel, and were placed into a polyethylene bag to prevent contamination. The experimental FME, transported to the ISS by carriers organized by NASA, was unclamped twice by astronauts on the ISS: on the day of arrival on the ISS (day 25) and 14 days before the end of its ISS stay (day 50). A second, identical experiment was conducted on Earth as a control. Worms were harvested from both systems on day 70 after tube sealing. The space $C$. elegans spent a total of 40 days at microgravity. Table 1 shows a complete timeline of the experiment, including changes in location, temperature, and state of the FME during its round trip to the ISS and back.

Prior to the launch, we determined the optimal ratio of CeMM to air in each volume of the FME for maximum survival of $C$. elegans. We optimized our ratios by assembling FMEs containing variable volumes of CeMM and air and observed the worms to determine which conditions maximized survival. We observed the highest number of live $C$ elegans after 70 days using $2.4 \quad \mathrm{~mL} \quad$ CeMM/compartment (2.1 worms $/ \mu \mathrm{L})$, compared to $2 \mathrm{~mL}$ CeMM/compartment (2.5 worms $/ \mu \mathrm{L}$ ), and $2.8 \mathrm{~mL}$ CeMM/compartment (1.8 worms $/ \mu \mathrm{L}$ ). Therefore, the optimal ratio of $2.4 \mathrm{~mL}$ of CeMM and $0.4 \mathrm{~mL}$ of air was selected, as this ensured that there were live nematodes in the FME throughout the tenweek experimental period. 
Table 1. Timeline of the experiment. The letter A stands for arrival (at the ISS) and the letter U stands for undock from the ISS.

\begin{tabular}{|c|c|c|c|c|}
\hline Location & Time Start & $\begin{array}{c}\text { Time } \\
\text { Duration }\end{array}$ & $\begin{array}{c}\text { Temperature } \\
\left({ }^{\circ} \mathrm{C}\right)\end{array}$ & $\begin{array}{c}\text { Changes made } \\
\text { to the FME }\end{array}$ \\
\hline $\begin{array}{c}\text { Travel from Toronto } \\
\text { to Houston (FedEx) }\end{array}$ & A-25 days & 2 days & $2-4$ & None \\
\hline $\begin{array}{c}\text { NanoRacks } \\
\text { (Houston) }\end{array}$ & A-23 days & 12 days & $2-4$ & None \\
\hline $\begin{array}{c}\text { Travel from Ferry to } \\
\text { Launch }\end{array}$ & A-11 days & 11 days & $2-4$ & None \\
\hline $\begin{array}{c}\text { Rocket travel to ISS } \\
\text { ISS }\end{array}$ & A-6 hours & 6 hours & $2-4$ & $\begin{array}{c}\text { A+0 days: Unclamp Clamp A, } \\
\text { shake gently for 3 seconds } \\
\text { A-14 days: Unclamp Clamp } \\
\text { B, shake gently for 3 seconds }\end{array}$ \\
\hline $\begin{array}{c}\text { Travel from Landing } \\
\text { to Houston }\end{array}$ & U & 4 days & $0-10$ & $21-24$ \\
\hline $\begin{array}{c}\text { Travel from Houston } \\
\text { to Toronto (FedEx) }\end{array}$ & U+4 days & 24 hours & $2-4$ & None \\
\hline
\end{tabular}

\section{C. elegans Viability and Morphometric Analyses}

Following return from space, the number of C. elegans in both the experimental and ground FMEs were counted. A small equivalent aliquot of worms was taken from each tube, viewed with a phase contrast microscope, and photographed. The length and cross-sectional area of live $C$. elegans were measured, using ImageJ software, in 40 randomly chosen individual worms from each sample. The average length and area of the worms were then calculated for both the space and ground worms.

\section{SDS PAGE and ASM Immunoblotting}

To create the $C$. elegans protein lysate, both tubes of worms were washed three times with M9 solution, once with phosphate buffered saline (PBS), and then re-suspended in $75 \mu \mathrm{L}$ of ice-cold lysis buffer (25 mM Tris [pH 7.4], $100 \mathrm{mM} \mathrm{NaCl,}$ $1 \mathrm{mM}$ EDTA, 0.25\% NP40, $1 \mathrm{mM}$ PMSF, $1 \mathrm{mM}$
$\mathrm{Na}_{3} \mathrm{VO}_{4}, 2.5 \mathrm{mg} / \mathrm{mL}$ Pepstatin-A, $10 \mathrm{mM} \mathrm{NaF}$, and 1 Protease Inhibitor Cocktail Tablet [Roche, Basil, Switzerland] per $10 \mathrm{~mL}$ solution). The samples were incubated on ice for 30 minutes, then subjected to three cycles of flash freezing in liquid nitrogen, partial thawing, and sonication for 5 seconds at 7W. Samples were then incubated at $4^{\circ} \mathrm{C}$ with agitation for 30 minutes and centrifuged at 13,000 rpm. Supernatant protein concentration was determined with the BCA assay (Thermo Scientific, Waltham MA, USA).

Worm lysate samples (30 $\mu \mathrm{g})$ were separated by $10 \%$ SDS-PAGE, transferred to nitrocellulose, Ponceau-S stained, and immunoblotted with rabbit anti-acid sphingomyelinase antibody (sc-11352 Santa Cruz, CA, USA; 1:500 dilution). Prepared samples were analyzed with IRDye $800 \mathrm{CW}$ fluorescent secondary antibody (Mandel Scientific, Guelph ON, Canada; 1:10,000 dilution). Quantification of the $\mathrm{Li}^{-\mathrm{Cor}^{\mathrm{tm}}}$ fluorescent signal was performed with the LiCor Odyssey FC (Mandel Scientific). Western blotting 
for GAPDH (ab A245 Abcam, USA; 1:10,000 dilution detected with Mandel Scientific IRDye $680 \mathrm{RD}, 1: 10,000$ dilution) was performed as a loading control. Samples were run in duplicate.

\section{Statistical Analysis}

Comparisons between the space worms and control worms were conducted using the Student's t-test. Statistical significance was determined if $\mathrm{p}<0.05$.

\section{RESULTS}

\section{C. elegans Size and Morphometrics}

The space worms were received at the lab 70 days after initial loading into the FME. There were 72,050 live space worms, compared to 95,200 live ground worms. As illustrated in Figure 2, the space worms were significantly larger in length and in cross-sectional area than the ground worms. The mean length of the space worms was $0.42 \pm 0.13 \mathrm{~mm}$, while the ground worms exhibited a mean length of $0.29 \pm 0.076$ $\mathrm{mm}$. The mean cross-sectional area of the space worms was $0.0065 \pm 0.0031 \mathrm{~mm}^{2}$, while the mean cross-sectional area of the ground worms was only $0.0042 \pm 0.0021 \mathrm{~mm}^{2}$. The histograms in Figure 3 demonstrate a broader range of distribution of length and cross sectional area for the space worms compared to the control ground worms.

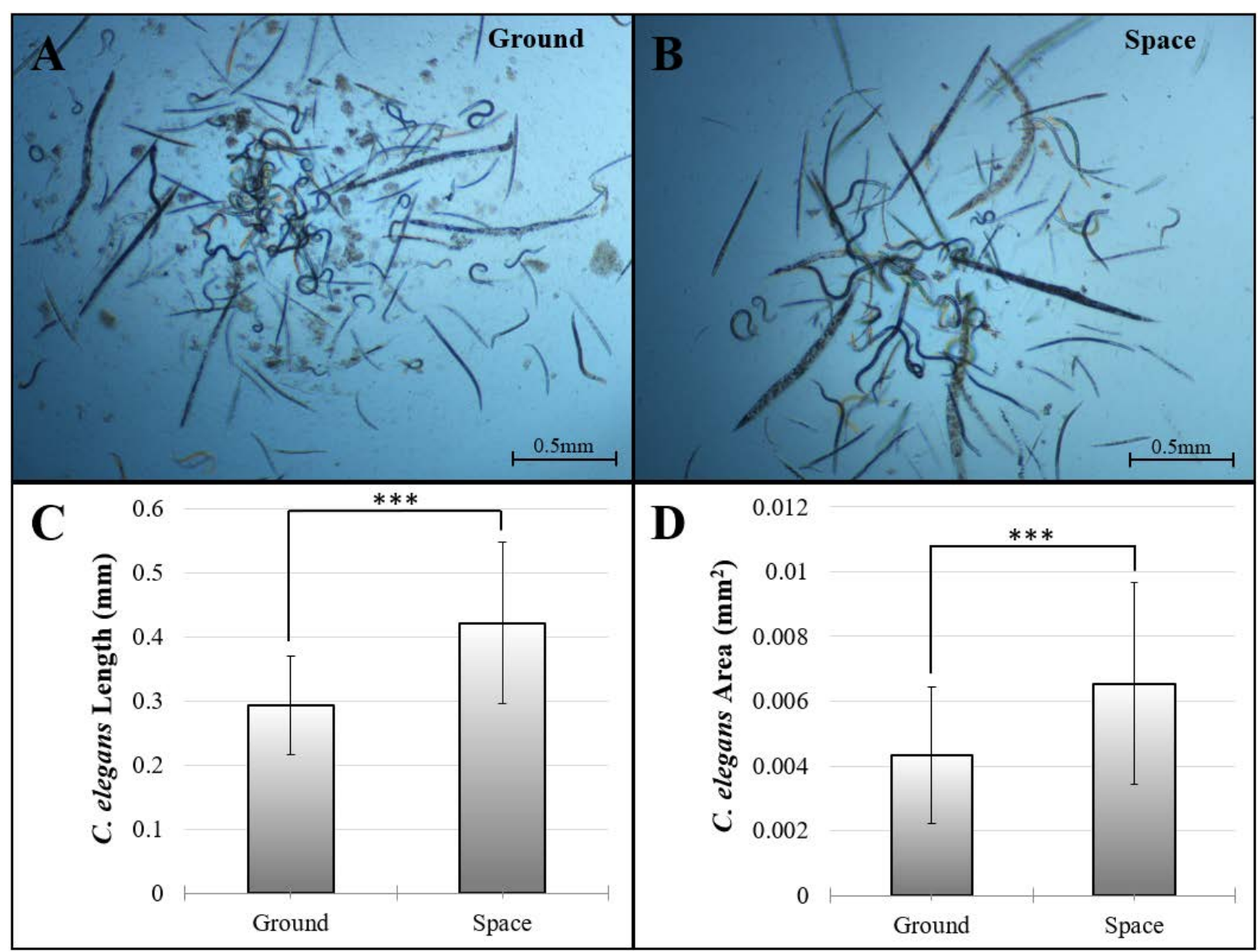

Figure 2. Length and cross-sectional area (CSA) of ground and space worms. Representative images of (A) ground worms, and (B) space worms are shown. Live $C$. elegans appear curled and dead $C$. elegans assume a straight and thick shape. Only live $C$. elegans were measured. The space worms $(\mathrm{N}=40)$ were longer $(\mathrm{C})$, and had larger CSA (D), compared to the control worms $(\mathrm{N}=40)$. Data are mean $\pm \mathrm{SD} . * * * \mathbf{p}<0.05$. 

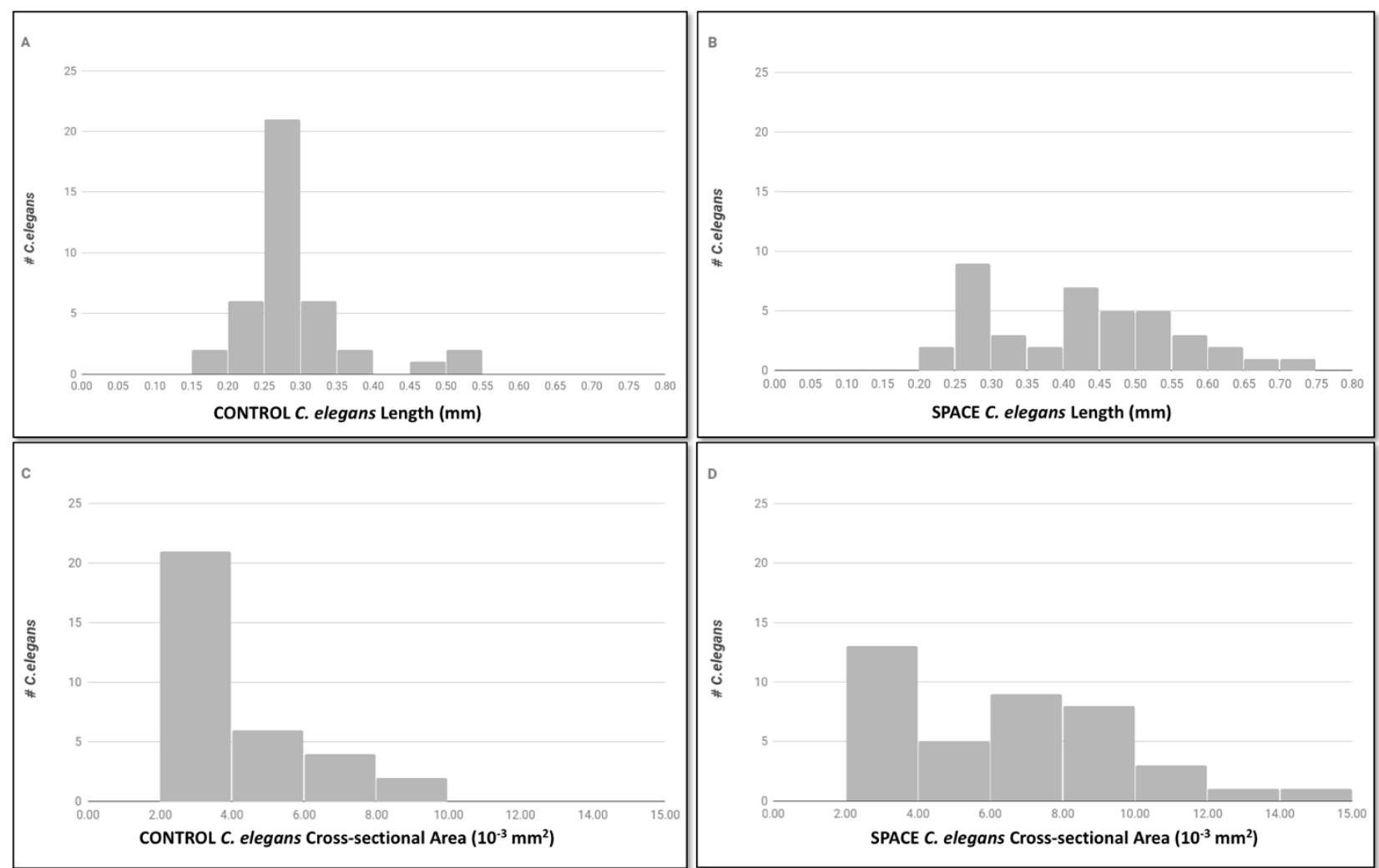

Figure 3. Histograms of length and cross-sectional area (CSA) of space and control C. elegans. The top panels depict the distribution of lengths of randomly selected control $C$. elegans $(n=40$, left panel) and space C. elegans $(n=40$, right panel). Bottom panels similarly demonstrate the distribution of CSAs of control (left panel) and space (right panel) worms. A broader distribution of both length and CSA is evident in the space worms compared to the control ground worms.

\section{ASM Levels}

As illustrated in Figure 4, the space worms exhibited reduced ASM-1 and ASM-2 expression, compared to the ground worms.

The space worm lysate had a higher protein concentration, compared to the ground worm lysate, as determined by a BCA assay (Thermo Scientific). The space worm lysate had a protein concentration of $6.667 \mu \mathrm{g} / \mathrm{mL}$, whereas the ground worm lysate had a protein concentration of $4.286 \mu \mathrm{g} / \mathrm{mL}$.

\section{DISCUSSION}

This is, to our knowledge, the first study completed to assess ASM protein expression in $C$. elegans in microgravity. The purpose of the experiment was to gain a better understanding of the molecular regulation of muscle atrophy in microgravity, which may also provide insight into the mechanisms of atrophy in disease. We hypothesized that oxidative stress and altered ASM expression are involved in the induction of muscle atrophy that results from both sustained exposure to microgravity, as is seen in astronauts, and from ALS. We predicted that ASM levels would be elevated in $C$. elegans exposed to microgravity, as sphingomyelinase gene expression levels and ceramides have been found to increase in mouse models of ALS (Henriques et al., 2018; Cutler et al., 2002) We also aimed to test whether the nematodes' muscle mass would be impacted by the microgravity conditions.

We found that $C$. elegans exposed to microgravity were longer, had greater crosssectional areas, and had markedly reduced ASM-1 and ASM-2 expression compared to the control worms. . These are striking and unanticipated results, contrary to our hypothesis and previous literature. Muscle atrophy is observed in humans in spaceflight and previous studies have shown 

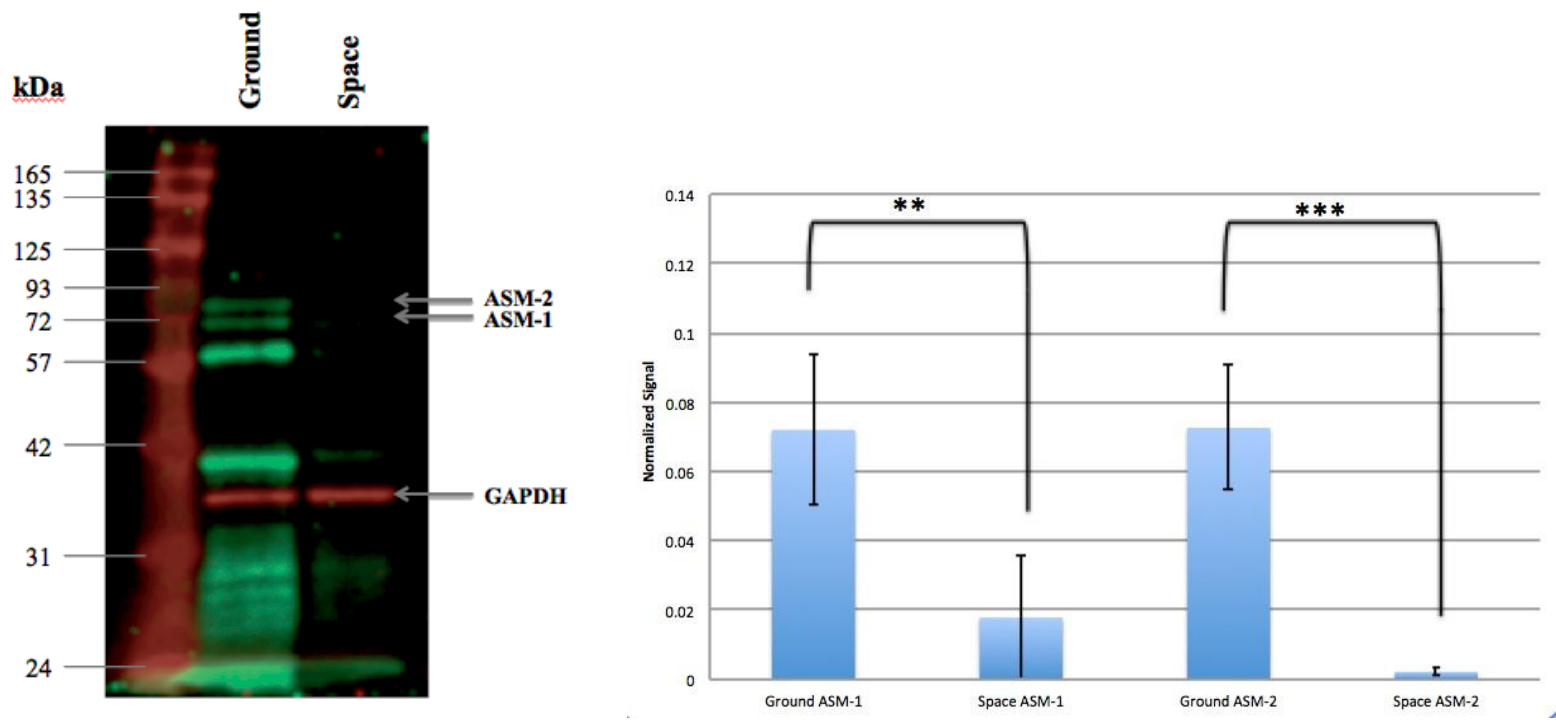

Figure 4. Ground and space worm lysates immunoblotted for ASM-1 and ASM-2. The space worms exhibited decreased expression of both ASM-1 and ASM-2, compared to the ground worms (left panel ASM Western blot, right panel graph of quantified fluorescent signal). GAPDH immunostaining demonstrates equivalent protein loading. Data are mean \pm SD. ${ }^{* * *} \mathbf{p}<0.05, * * \mathbf{p}<0.1$.

similarly that C. elegans in microgravity experience decreased rates of myogenic transcription and have lower muscle density (Higashibata et al., 2006). Higashibata (Higashibata et al., 2016) also reported a decrease in length and fat accumulation in the space flown C. elegans. Furthermore, ASM-2 gene expression analyses using cDNA microarrays from International C. elegans first experiment (ICEFIRST) (Selch et al., 2008) and C. elegans RNai space experiment (CERISE) (Higashitani et al., 2009) showed a 1.3 fold increase and a 1.1 fold decrease, respectively, although these results were not statistically significant (unpublished data, personal communication with Nathaniel J. Szewczyk).

Interestingly, Kim and Sun (2012) report that inactivation of the ASM homologs leads to slower reproduction and increased longevity of $C$. elegans. Given the lower expression levels of both ASM-1 and ASM-2 in the space worms, we speculate this may have increased their longevity, resulting in an increase in size compared to the control worms. Furthermore, slower reproduction meant that there were fewer worms in the space tube than in the control tube. As a result, each space worm had greater access to nutrients and oxygen, which might also explain why the space worms were able to grow larger. The reason for the decrease in ASM levels in space worms relative to the control worms is not known. ASM expression and activity is influenced by stress (Chung et al., 2016), and stressors may have impacted the two cohorts of worms differentially, but this was not assessed in our study.

Of interest, we were able to demonstrate that a population of $C$. elegans can survive for 10 weeks within the confines of a $17 \mathrm{~cm} \mathrm{FME;} \mathrm{to} \mathrm{our}$ knowledge this is a novel finding. A previous study completed as part of the SSEP program did not yield live C. elegans upon return to Earth, due to an error in feeding the worms during spaceflight. This experiment concluded that the FME could not produce viable populations for post-flight analysis on extended missions such as the SSEP missions (Warren et al., 2013). With the return of 72,050 live $C$. elegans from the ISS, our study demonstrates that nematode populations can survive with limited oxygen and nutrients for at least 70 days. Not only did the worms survive space travel, but the population grew to more than fourteen times its original size. Therefore, $C$. elegans may be used as a model organism for 
future prolonged microgravity experiments in FME tubes.

There were a number of limitations to our study, as a result of regulations set by NanoRacks, NASA, and SpaceX. Only one tube could be sent to space, thereby permitting no experimental replicates, which limited the power of our experiment. Although we could not send multiple FME tubes to the ISS, we maximized the number of $C$. elegans within the tube to account for variability between worms. Additionally, the experiment needed to be performed in an FME, which was difficult to use. It was prone to leakage, contamination, and overall wear and tear. Although preliminary testing conducted by us to optimize experimental conditions allowed us to determine the most effective way to seal, subtle leakage or contamination during spaceflight could have impacted our results. In order to prevent leakage, two twist ties, gel, and a plastic cap were placed on each end of the tube; however, $1.5 \mathrm{~mL}$ of liquid was still lost due to leakage during the trip.

There were also several limitations of the experimental design. We did not test for the third ASM isoform, ASM-3, which may also affect the lifespan of the nematodes and their oxidative stress levels. We did not test for ASM-3 because a commercially produced antibody was not available. The anti-ASM antibody used was generated against the human ASM, and while it recognized proteins at the correct molecular weight of ASM-1 and ASM-2, it also recognized several other bands of unknown significance. These bands may have been due to non-specific reactivity or reactivity with degradation products. It is unclear why these bands were only seen in the ground control worms, given that GAPDH levels indicated equal loading of ground and space worm lysates. Furthermore, we did not evaluate oxidative stress, ASM-1 or 2 activity, or evaluate behavioral changes in the worms, which may be influenced by oxidative stress and could have served as a surrogate marker (Possik and Pause, 2015). Although this data may have enriched our knowledge, we were mainly focused on the impacts of microgravity on ASM expression. Lastly, C. elegans size is in part dependent on maturation stage. Notably we did not evaluate maturation stage of the space or ground worms, which may have confounded our morphometric measurements.

In summary, as high school students working within the SSEP program, we have importantly demonstrated that $C$. elegans can survive for at least 70 days with limited nutrients, space, and oxygen and are therefore a feasible and effective model for spaceflight and for SSEP experiments. We also demonstrated a statistically significant difference in expression of ASM between ground and space worms, although the underlying mechanisms are not known. Future research should aim to further evaluate the role of ASM in muscle atrophy in both spaceflight and in diseases such as ALS, as this may enable the identification of targets and strategies for therapeutic manipulation.

\section{ACKNOWLEDGEMENTS}

First and foremost we thank the Student Spaceflight Experiment Program for this extraordinary opportunity. We would like to acknowledge the contributions of the rest of the team at the University of Toronto Schools (Michael Liu, Catherine Vlasov, Miguel RicoLuengo, Wilson Ho, and Maddie Elder) for their help in the initial stages of the project. We would like to thank the scientists who supervised our experiments and/or provided advice in experimental design, data analysis, and laboratory equipment/space: Dr. Robert D’Cruz, Dr. Jane Batt, Judy Correa, Dr. Peter Roy, May Yeo, Dr. Nathaniel Szewczyk, and Dr. Lew Jacobson. We express our sincere gratitude to our mentor, Suzanne Monir, for her support, guidance, and spearheading project funding campaigns. We would also like to thank Mr. Shawn Brooks for supporting the project. We also thank Dr. Luis Rene Garcia and his student Liusuo Zhang for providing us with the CeMM for the experiment.

\section{REFERENCES}

Adenle AA, Johnsen, B, Szewczyk NJ (2009) Review of the results from the International C. elegans first experiment (ICE-FIRST). Advances in Space Research 44(2): 210216

Altun ZF, Hall DH (2006) Introduction to $C$. elegans anatomy. In WormAtlas. http://www.wormatlas.org/ver1/handbo 
ok/anatomyintro/anatomyintro.htm

(Accessed June 20, 2018)

Avery L, You Y (2012) C. elegans feeding. In WormBook (ed. The C. elegans research community)

Brooks NE, Myburgh KH (2014) Skeletal muscle wasting with disuse atrophy is multidimensional: the response and interaction of myonuclei, satellite cells and signaling pathways. Frontiers in Physiology 5: 99

Chung HY, Hupe DC, Otto GP, Sprenger M, Bunck AC, Dorer MJ, Bockmeyer CL, Deigner HP, Graler MH, Claus RA (2016) Acid sphingomyelinase promotes endothelial stress response in systemic inflammation and sepsis. Molecular Medicine 22: 412-423.

Cutler R, Pederson W, Camandola S, Rothstein J, Mattson M (2002) Evidence that accumulation of ceramides and cholesterol esters mediates oxidative stress-induced death of motor neurons in amyotrophic lateral sclerosis. Annals of Neurology 52: 448-457

Fanzani A, Conraads VM, Penna F, Martinet W (2012) Molecular and cellular mechanisms of skeletal muscle atrophy: an update. Journal of Cachexia, Saropenia and Muscle 3: 163-179

Fitts RH, Riley DR, Widrick JJ (2001) Functional and structural adaptations of skeletal muscle to microgravity. Journal of Experimental Biology 204: 3201-3208

Fitts RH, Trappe SW, Costill DL, Gallagher PM, Creer AC, Colloton PA, Peters JR, Romatwoski JG, Riley DA (2010) Prolonged space flight-induced alterations in the structure and function of human skeletal muscle fibres. The Journal of Physiology 588(Pt 18): 3567-3592

Henriques A, Croixmarie V, Bouscary A, Mosbach A, Keime C, Boursier-Neyret C, Walter B, Spedding M, Loeffler JP (2018) Sphingolipid metabolism is dysregulated at transcriptomic and metabolic levels in the spinal cord of an animal model of amyotrophic lateral sclerosis. Frontiers in Molecular Neuroscience 10: 433

Higashibata A, Hashizume T, Nemoto K, Higashitani $\mathrm{N}$, Etheridge $\mathrm{T}$, Mori C, Haranda S, Sugimoto T, Szewcyzk NJ,
Baba SA, Mogami Y, Fukui K, Higashitani A (2016) Microgravity elicits reproducible alterations in cytoskeletal and metabolic gene and protein expression in space-flown Caenorhabditis elegans. npj Microgravity 2: 15022

Higashibata A, Szewczyk N, Conley C, ImamizoSato M, Higashitani A, Ishioka N (2006) Decreased expression of myogenic transcription factors and myosin heavy chains in Caenorhabditis elegans muscles developed during spaceflight. Journal of Experimental Biology 209: 3209-3218

Higashitani A, Hashizume T, Sugimoto T, Mori C, Nemoto K, Etheridge T, Higshitani N, Takanami T, Suzuki H, Fukui K, Yamazaki T, Ishioka N, Szewczyk N, Higashibata A (2009) C. elegans RNai space experiment (CERISE) in Japanese Experiment Module KIBO. Biological Sciences in Space 23(4): 183-187

Jana A, Hogan E, Pahan K (2009) Ceramide and neurodegeneration: Susceptibility of neurons and oligodendrocytes to cell damage and death. Journal of Neurological Science 278(1-2): 5-15

Kim Y, Sun H (2012) ASM-3 acid sphingomyelinase functions as a positive regulator of the DAF-2/AGE-1 signaling pathway and serves as a novel anti-aging target. PLOS ONE 7(9): e45890

Kornhuber J, Rhein C, Müller CP, Mühle C (2015) Secretory sphingomyelinase in health and disease. Journal of Biological Chemistry 396(6-7): 707-36

Lin X, Hengartner M, Kolesnick R (1998) Caenorhabditis elegans contains two distinct acid sphingomyelinases. Journal of Biological Chemistry 272(23): 14374 14279

Loeffler JP, Picchiarelli G, Dupuis L, De Aguilar JL (2016) The role of skeletal muscle in Amyotrophic Lateral Scleosis. Brain Pathology 26: 227-236

Possik E, Pause A (2015) Measuring oxidative stress resistance of Caenorhabditis elegans in a 96 well microtiter wells. Journal of Visualized Experiments 99: 52746

Powers SK, Lynch GS, Murphy KT, Reid MB, Zijdewind I (2016a) Disease-induced muscle atrophy and fatigue. Medicine \& 
Gravely et al. -- Levels of ASM in C. elegans in Microgravity

Science in Sports \& Exercise 48(11): 23072319

Powers SK, Morton AB, Ahn B, Smuder AJ (2016b) Redox control of skeletal muscle atrophy. Free Radical Biology and Medicine 98: 208-217

Selch F, Higashibata A, Imamizo-Sato M, Higashitani A, Ishioka N, Szewczyk NJ, Conley CA (2008) Genomic response to the nematode Caenorhabditis elegans to spaceflight. Advances in Space Research 41(5): 807-815

Szewczyk NJ (2005) Caenorhabditis elegans survives atmospheric breakup of STS-107, space shuttle Columbia. Astrobiology 5(6): 690-705

Szewczyk NJ, Jacobson LA (2005). Signaltransduction networks and the regulation of muscle protein degradation. International Journal of Biochemistry and Cell Biology 37(10): 1997-2011.
Takahashi K, Okumura H, Guo R, Naruse K (2017) Effects of oxidative stress on the cardiovascular system in microgravity. Journal of Molecular Science 18: 1426

Tanaka K, Nishimura N, Kawai YJ (2017) Adaptation to microgravity, deconditioning and countermeasures. The Journal of Physiological Sciences 67: 271-281

van Es MA, Hardiman O, Chio A, Al-Chalabi A, Pasterkamp RJ, Veldink JH, van den Berg LH (2017) Amyotrophic lateral sclerosis. Lancet 390: 2084-98

Warren P, Golden A, Hanover J, Love D, Shephard F, Szewczyk N (2013) Evaluation of the fluids mixing enclosure system for life science experiments during a commercial Caenorhabditis elegans spaceflight experiment. Advances in Space Research 51(12): 2241-2250 